\title{
EXPLORING THE DYNAMIC INTEGRATION OF HETEROGENEOUS SERVICES
}

\author{
Makaziwe Makamba, Jabu Mtsweni and Ernest Ketcha Ngassam \\ ${ }^{1}$ Department of Computer Engineering, \\ University of South Africa, Pretoria, South Africa \\ 55423434 @mylife.unisa.ac.za \\ ${ }^{2}$ CSIR, Meiring Naude Road, Pretoria, South Africa \\ makamba.makaziwe93 @gmail.com
}

\begin{abstract}
The increase need for services to handle a plethora of business needs within the enterprise landscape has yielded to an increase in the development of heterogeneous services across the digital world. In today's digital economy, services are the key components for communication and collaboration amongst enterprises internally and externally. Since Internet has stimulated the use of services, different services have been developed for different purposes prompting those services to be heterogeneous due to incompatibles approaches relied upon at both conceptual and exploitation phases. The proliferation of developed heterogeneous services in the digital world therefore comes along with a range of challenges more precisely in the integration layer. Traditionally, integration is achieved by using gateways, which require considerable configuration effort. Many approaches and frameworks have been developed by different researchers to overcome these challenges, but up to date the challenges of integration heterogeneous services with minimal user-involvement still exist. In this paper, we are exploring the challenges of heterogeneous services and characteristics thereof with the aim of developing a seamless approach that will alleviate some of these challenges in near future. It is therefore of outmost importance to understand the challenges and characteristics of heterogeneous services before developing a mechanism that could eliminate these challenges.
\end{abstract}

\section{KEYWORDS}

Integration, heterogeneous, dynamic-integration, services, and heterogeneous-services.

\section{INTRODUCTION}

The rapid evolution of internet connectivity and service enterprises has raise potential interaction with many other service enterprises across the globe. In this context, the services are defined as an abstracted, logical view of actual programs that exchange messages between provider agent and requester [1]. A service is also defined as an abstract resource that represents a capability of performing tasks that form a coherent functionality [2]. The key to these services is loosely coupled nature, where the service interface is independent of the implementation. The increasing

David C. Wyld et al. (Eds) : CSEN, SIPR, NCWC - 2016

pp. 103-110, 2016. C CS \& IT-CSCP 2016

DOI : $10.5121 /$ csit.2016.61009 
number of heterogeneous services has made a significant drawback on the scalability, integration and the performance of the services [3]. Heterogeneous service in this context is defined as a uniqueness of services that are developed on different environments using different techniques and architectures to complete a specific purpose. These services have to interact with each other to achieve a certain business goal. As the development of services is increasing in enormous way across the domain, there is a need to find appropriate approach that will allow these heterogeneous services to be dynamically integrated without using hard-wiring approaches, which are tiresome [3]. There are traditional methods of integrating these heterogeneous services; however these traditional methods are not adequate as the require user-intervention when there is a change in service that need to be integrated. The traditional methods have some problems, such as the code complexity more difficult to maintain, lead the efficiency of the bottleneck easily [4]. It is therefore, essential to develop a method that will enable dynamic integration of the heterogeneous services without the need for user-intervention.

This paper is focusing on identifying the challenges of heterogeneous services integration; describe the characteristic of heterogeneous services and discussing the advantages and limitations of the existing integration models.

The remainder of the paper will discuss the heterogeneous services integration in Section 2 . Section 3, will discuss the characteristics of heterogeneous services, the challenges of heterogeneous services which is the core of this paper will be discussed in Section 4 of this paper. The related work on what other researchers developed to alleviate these challenges will be discussed in section 5. Section 6 will conclude the paper with the recommendations of what needs to be developed to eliminate these challenges.

\section{HETEROGENEOUS SERVICE INTEGRATION}

Most of the services are heterogeneous, which means they are executed under different platforms, designed using different architectures and programming languages [5]. Heterogeneous services are services that are developed by diverse vendors for different purposes on different platforms, using different architectures; different protocols and different languages [6]. These heterogeneous services need to be integrated. Heterogeneous service integration is not a new phenomenal in the field of integration. Integrating heterogeneous services in a dynamic manner may improve if it could include prescriptive or solution-oriented knowledge where the result from scientific justification (predicting, understanding or explaining phenomena) can be used in designing solutions to this complex problems [7].

However, heterogeneous service integration has become major issue in the area of integration. This is motivated by complexity of these heterogeneous services and independent services when they need to communicate with one another to achieve a specific business goal. Service integration has to be performed for these services to communicate.

Service integration in this context is defined as the integration of discrete IT services components into a coherent set of end-to-end service bound by Operation Level Agreements (OLAs) and Services Level Agreements (SLAs) [9]. The service integration is a challenge when these heterogeneous services need to interact. There are certain aspects that make these services to be heterogeneous, which results to challenge when these services need to be integrated. The following section will discuss in details the characteristics of heterogeneous services. 


\section{HeTEROGENEOUS SERVICES CHARACTERISTICS}

Inter-enterprise integration is an essential requirement for today's successful business [10]. With the aim of overcoming heterogeneity, various technologies and standards for the definition of languages, vocabularies and integration patterns are being developed. However, before discussing the pattern and the technologies that have been developed, there is a need to define the heterogeneous services and the characteristic of these services. As the above section has defined the heterogeneous services, this section will discuss the characteristics of these heterogeneous services. After an extensive literature review and the empirical study that was conducted. We have discovered that services are heterogeneous based on the following aspects; environment, technology, architecture and programming language [11]. As Hajiji mentioned in 2012, there are many fundamental aspects that synthesize the heterogeneity in these services [12]. The heterogeneity of services is also characterized by 4 fundamental aspects.

1. Environment- Due to execution environment, as some services are developed to be executed on different environments such as Mobile Services, Cloud Services, Desktop services, Web Services, ATM Services and many more. These services are explicitly developed to run on these environments for a specific purpose.

2. Systems and Technology- There are several services that run on specific system. Some services are developed to run on Android, some on IOS, while some run on Linux, Windows and Mac OS. Systems and technology are making these services heterogeneous.

3. Architecture- Architecture is another fundamental aspect that differentiates services. Some services are developed for Service Oriented Architecture (SOA) and some on Enterprise application Integration (EAI) while some other architecture allows services to return a JSON and while some are allowed to return Extensible Mark-up Language (XML).

4. Programming Language- This is another component that makes services to be heterogeneous. Some services are developed using php while others are developed using Java, C++ and C\#. This makes services to be heterogeneous as they are difficult to integrate due to different programming languages [9].

These above characteristics are granting a challenge on service integration environment. As the figure 1 below depicts, services can be heterogeneous because of the deployment environment, could be mobile, desktop or laptop, and the architecture that is used to develop the services. However these services can be heterogeneous but they need to talk to each other to share information.

As it shown in the picture, these services are deployed in different environment; they are using different architecture and different technology. There are services that are deployed for mobile phone, there are services that are created for desktops and there are services are designed for laptops, however these service needs to interact with each other. It is therefore critical to integrate these services in a dynamic manner. A manner that will not require user-intervention, as it delays the progress of integration task. In the following section, this paper will discuss in details the key challenges of heterogeneous service integration. 


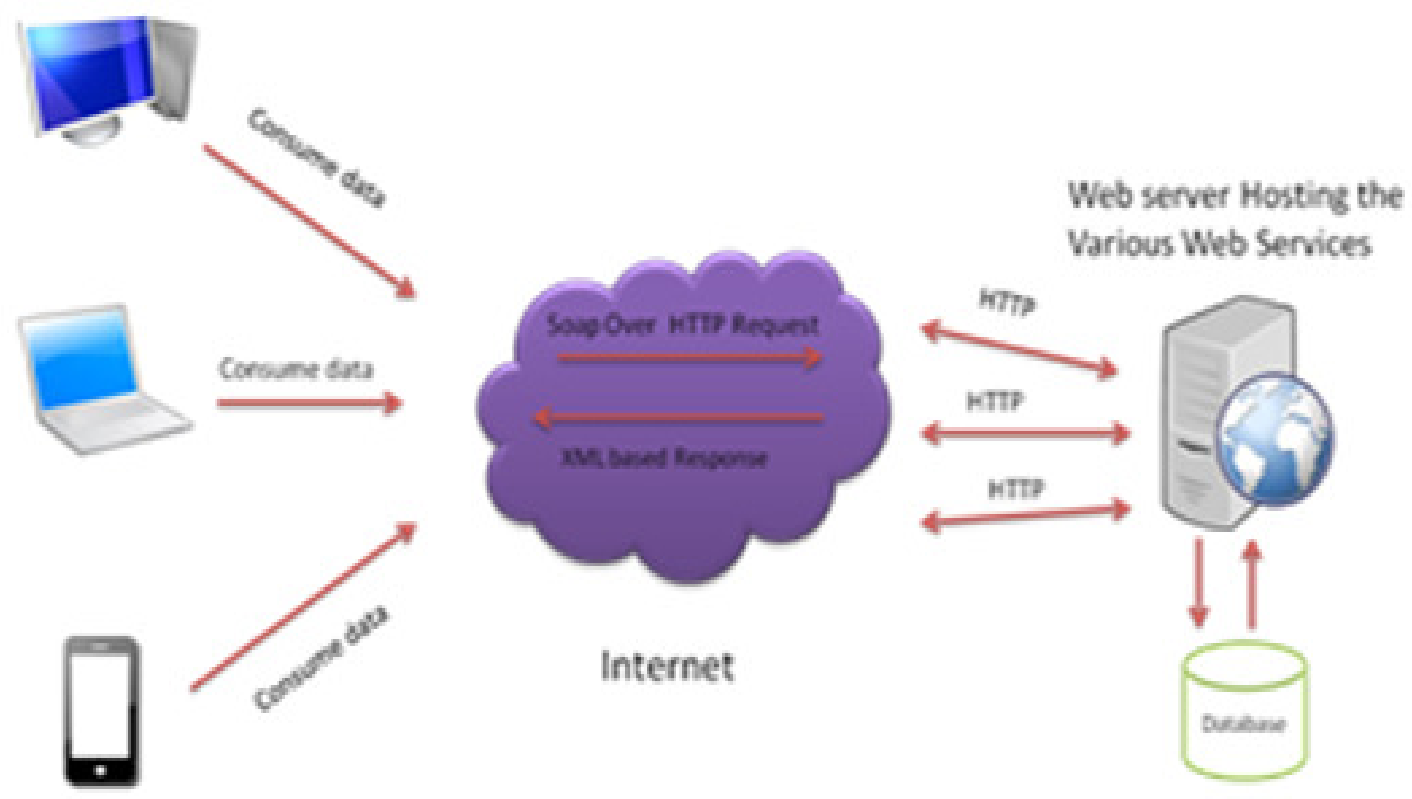

Figure 1: Heterogeneous Service Integration [9]

\section{CHALlenges OF HeTEROGENEOUS SERVICE INTEGRATION}

In today's world, many enterprises use services as a main core of communication. The challenge that most organizations are facing is to provide a method that could allow dynamic integration of heterogeneous service, a method that can allow these services to work together to address business goals that constantly evolve [13]. There are several heterogeneous services that have been developed by different vendors, such as mobile services, cloud services, web services and others [14]. Most of these services are integrated manually by using Application programming interface (APIs), Adapters, ESBs and many more. This led the companies to lose profit in their business as the integration task becomes cumbersome for the developer, as he/she needs to be involved when integrating these services. These services has varied and multiple integration points. This Increases the challenge of integrating heterogeneous services on the fly. Each service is independent and performs its own task, which might be valuable to a cohesive system. The other challenge that makes it difficult for integration is multiple data models and multiple instances of the services. Achieving a seamless flow of information requires a significant integration method. A method that can scale with business needs as new applications and platforms appear. The current methods of integrating heterogeneous services are very tedious, inflexible and time consuming when the services change requirements [14].

One of the aspects that make these services to be complex to integrate is the 4 mentioned fundamental characteristics that are mentioned above. As these services run on different platform, environment, and system and developed using different programming languages. It makes it difficult to integrate them as they are heterogeneous. Most of the services are currently integrated using manual approach which is to develop an API for each service that needs to be integrated. When there is a change on a service an API has to be modified which make it unmanageable when there is increase in number of services. Hence this manual method is regarded as an inadequate method [14]. 
Integrating heterogeneous services in order to connect their functionality is utmost importance for promoting deployment dynamic integration of heterogeneous service. It is therefore important to understand the characteristic of these heterogeneous services and explore the challenges of integrating them in order to develop a mechanism to that will enable dynamic integration of these services. A method that will allow heterogeneous services to integrated with a minimal userinvolvement. Hence we are exploring the challenges so that we can develop a method that will alleviate these challenges in near future.

Many studies have been conducted to resolve the issue of heterogeneous service integration. The following section will discuss more in details about the related work.

\section{RELATED WORK}

The challenges of heterogeneous service integration is not a new phenomenal in the field of integration. Many researchers have explored the different paradigm to develop solutions that can eliminate these challenges of heterogeneous service integration. However, up to today, the challenges have not been resolve completely. A new service integration system was developed by Huiyang (2006) to resolve the existing challenges. This system only allows the integration of services at a component level [7]. In this system a user can insert common service logic to their business logic [7]. The system establishes an agent between service components; this was done to allow the request and orchestration to identify the components in a correct manner [7]. However, this system requires a developer's intervention to integrate, modify and change certain service requirements.

Another agent-based web service integration model was developed by $\mathrm{Yu}$, et al (2008) to integrate the services and ensuring the Quality of Services (QOS) of the integration system [8] This QOS-based integration was developed to improve services integration efficiency and minimize the integration cost through the selection mechanism. However, this mechanism worked for integrating services but it does not eliminate the user-involvement challenge when the change is required on a services. Another integrated model was proposed and the integration framework of component-based was constructed by Shaoba et al (2010) to solve the problems of heterogeneous service integration [4]. This integration model was done for user management and user access control and register service component. This framework was mainly used to standardize enterprise business processes, to coordinate the data processing, and to build configurable enterprise service bus; data management modules [4].This framework has achieved data synchronization between heterogeneous systems but has not solved the problem of heterogeneous service integration with a minimal user-involvement. Hence, there is a need to develop a mechanism that will dynamically integrate heterogeneous services without a need for user-intervention. The following table will give more advantages and limitations of the current methods of integration. 
Table1: Comparisons of integration models

\begin{tabular}{|c|c|c|}
\hline Integration model & Advantages & Limitations \\
\hline ESB & $\begin{array}{l}\text { Services are connected to this logical } \\
\text { bus through smart connectors, which } \\
\text { encapsulate system functionality } \\
\text { and provide a layer of abstraction } \\
\text { between bus and application. } \\
\text { ESB use of open communication } \\
\text { standards, connectivity between bus } \\
\text { and applications is established. } \\
\text { ESB uses several integration points. } \\
\text { It's easy to onboard new services and } \\
\text { new nodes when using ESB. }\end{array}$ & $\begin{array}{l}\text { Requires ongoing management of } \\
\text { message versions to ensure the intended } \\
\text { benefit of loose coupling. Though ESB } \\
\text { systems can require a significant effort } \\
\text { to implement, they produce no } \\
\text { commercial value without the } \\
\text { subsequent development of SOA } \\
\text { services for the ESB. ESB have lot of } \\
\text { upfront overheads. When using ESB } \\
\text { there is a need to define canonical } \\
\text { message format. } \\
\text { The developmental complexity. } \\
\text { It normally requires more hardware } \\
\text { than simple point-to-point messaging. } \\
\text { ESB becomes a single point of failure. } \\
\text { It requires analysis skills to configure, } \\
\text { manage and operate ESB. }\end{array}$ \\
\hline EAI & $\begin{array}{l}\text { EAI solutions provide process } \\
\text { management functionality to } \\
\text { orchestrate inter-application message } \\
\text { exchanges, and an administration } \\
\text { console to monitor and track the } \\
\text { workings of the hub. Maintains } \\
\text { information integrity across multiple } \\
\text { systems. EAI focuses on sharing both } \\
\text { business data and business process. } \\
\text { EAI focus on integrating services on } \\
\text { the data level, application level and the } \\
\text { method level. }\end{array}$ & $\begin{array}{l}\text { EAI is more on point-to-point effort, } \\
\text { which becomes unmanageable as } \\
\text { number of services increases. } \\
\text { EAI consume considerable time and } \\
\text { effort to deploy, and are subject to high } \\
\text { project failure rates. } \\
\text { Requires expect knowledge to integrate } \\
\text { services using EAI. } \\
\text { Require a fair amount of up front } \\
\text { design, which many managers are not } \\
\text { able to envision. }\end{array}$ \\
\hline
\end{tabular}




\begin{tabular}{|c|c|c|}
\hline Middleware & $\begin{array}{l}\text { Middleware enable services to } \\
\text { exchange messages, even though we do } \\
\text { not know the platform of other service. } \\
\text { It guarantees throughput, reliability and } \\
\text { efficiency. } \\
\text { It flexible to modify and separate } \\
\text { interfaces from the applications. } \\
\text { Independence of layers and database. } \\
\text { Transparency and it easily customize } \\
\text { all components. }\end{array}$ & $\begin{array}{l}\text { It uses API to integrate services. } \\
\text { User-intervention is required whenever } \\
\text { there is a change in services that need } \\
\text { to be integrated. It is limited in terms of } \\
\text { scalability, each client adds overhead. It } \\
\text { uses low-level languages. } \\
\text { A small change is the environment } \\
\text { development can lead to major } \\
\text { downtime of real-time applications. It } \\
\text { is expensive. }\end{array}$ \\
\hline
\end{tabular}

Due to these series of limitations on the existing models of integration, this is a reason why there is a need to develop a method that will enable dynamic integration of heterogeneous service. Due to advantages of dynamic integration model, it would be easy to integrate heterogeneous service without a need of user-intervention. This could eliminate the challenges that many business enterprise are facing. As we can identify the gap that needs to be closed, dynamic integration could be a solution to this existing challenge. Dynamic integration would be a proper solution because it does not need user-intervention. It does not need a developer to modify API as the services will automatically update them. It would be scalable since services are integrated on the fly. In these days is hard to live normally without having to rely on some kind of technology. Technology is everywhere and used in different ways by all kinds of people in different work areas. Enterprises are now exposing their business as services for global visibility and for automation of business processes [15]. It is important to understand the current challenges and what has been developed to eradicate this challenge. In future we will develop a dynamic method of integrating heterogeneous service without a need of user-intervention.

\section{CONCLUSION}

The challenges of heterogeneous service integration are affecting multiple organizations. The technology must be flexible and scalable in reconciling semantic differences amongst these information exchange entities. It is therefore essential to have mechanisms that will dynamic integrate heterogeneous services, to make integration as seamless as possible. Hence in this paper, we described the challenges, characteristics of integration heterogeneous services, and comparison of existing models of integration so that it can be easy to develop a method that will close the existing gaps. In future we will develop a method that will allow dynamic integration of these heterogeneous services.

\section{ACKNOWLEDGMENT}

This work is undertaken within the Council for Scientific and Industrial Research (CSIR) in Defense, Peace Safety and Security (DPSS). The support from University of South Africa (UNISA) is also acknowledged. 


\section{REFERENCES}

[1] A. Reshamwala, G. Khetan and K. Gupta, (2009) "AIDS Helpline based on Service Oriented Architecture"

[2] H. Zhu, E. S, Madnick, (2004) "Context interchange as a Scalabe Solution to Interoperating Amongst Heterogeneous Dynamic Services"

[3] W. He, and L.H. Xu. (2011) "Integration of Distributed Enterprise Applications. IEEE procedengs.

[4] L. Shaobo, H.Yao and X. Qingsheng,(2010) "Heterogeneous system integration based on service component" Applied Mechanics and Materials Vols. 20-23 (2010) pp1305-1310.

[5] M.Makamba, (2016) "Preliminary Investigation of Challenges in Dynamic Integration of Heterogeneous Services" systemic, cybernetics and informatics volume 14-number1-year 2016, ISSN: $1690-4524$.

[6] Capgemini, (2013) "Service Integration; A blueprint for regaining control of complex IT vendor landscape" capgemini.

[7] X. Huiyang, S. Meina and S. Junde, (2006) "A new service integration system for modern service industry based on SOA" Proceedings of International conferences of technology and system in Beijing.

[8] W. Yu, (2011) "An integrated middleware based solution for supporting secured dynamic coating applications in Heterogeneous environment. IEEE, 2011.

[9] F. Hajaji, (2012) "Five Aspects of Application Integration Requirements. ARPN journal of system and software, 2012. Vol.2 no3

[10] Athanasopoulos.G, T. a. P., (2010) "Interoperability and Heterogeneous Services". China, MCSIN

[11] WebLogic, (2012) “Determining Integration Solution Requirements”, USA: BEA systems

[12] Makamba, M., Mtsweni, J. \& Ngassam, E. K., (2015) "Dynamic Integration of heterogeneous Services". Cape Town, South Africa, SAICSIT.

[13] MagnetyzingSolution, 2007. Middleware, India: Magnetyzing solutions

[14] Kaviani, N., Mohabbati, B. \& Lea, R., 2012. ReCoIn: A Framework for Dynamic Integration of Remote Services into a Service-Oriented Component Model. Colombia, ACM.

[15] Laliwala, Z., Kholsa, R., Majumdar, P. \& Chaudhary, S., (2006) "Semantic and Rules Based EventDriven Dynamic Web Services Composition for Automation of Business Processes". Chicago, USA, IEEE

\section{AUTHOR}

Makaziwe Makamba is a $\mathrm{PhD}$ candidate at the University of South Africa. She completed her Masters in 2012 at the University of Fort Hare in Alice, South Africa. Her area of interest is service integration, e-services and the dynamic integration.

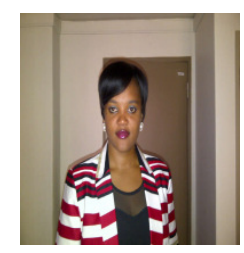

